\title{
A special issue on silicon and silicon-related materials for thermoelectricity ${ }^{\star}$
}

\author{
Dario Narducci ${ }^{\mathrm{a}}$ \\ University of Milano Bicocca, Department of Materials Science, via R. Cozzi 55, 20125 Milano, Italy
}

Received 10 June 2015

Published online 6 July 2015 - (c) EDP Sciences, Società Italiana di Fisica, Springer-Verlag 2015

Over the last years interest on thermoelectricity for microharvesting or for prospective large-scale conversion of heat into electric power has revamped. This was mostly due to the tremendous impact of nanotechnology onto the conversion rate achievable by thermoelectric generators [1], grown up by almost a factor three over the last two decades. Novel classes of thermoelectric materials have appeared - but also old materials have rejuvenated. Nanowires and nanolayers, along with multilayered structures, have imparted new improved characteristics to many age-old materials [2-4]. Two avenues were actually explored, namely reducing the thermal conductivity $\kappa$ and increasing the power factor $\sigma \alpha^{2}$ (where $\sigma$ is the electrical conductivity and $\alpha$ is the Seebeck coefficient). Both approaches lead to an increase of the thermoelectric figure of merit $Z T=\sigma \alpha^{2} T / \kappa$ (where $T$ is the absolute temperature) - and thus of the thermoelectric efficiency with which heat is converted into electric energy [5]. As thermoelectric performances have remarkably enhanced, much attention is now dedicated to selecting materials and technologies that could be scaled up and brought to bulk production. On this avenue, geo-abundance and low material costs are key issues. Thus, it is not surprising that thermoelectric properties of silicon, silicon-germanium alloys, and silicides had been the subject of more than 700 publications over the last five years. This Special Issue is devoted to cover the most important advances in this field through some mini-review papers and a selected number of research contributions, spanning from theoretical and experimental research on silicon-based nanostructured materials to the technology enabling their implementation in actual thermoelectric generators.

Possibly, a key date in the history of nanostructured thermoelectric silicon may be set at 2008, when Nature published back-to-back two seminal papers by Hochbaum et al. [6] and by Boukai et al. [7] on nanowires. Both

\footnotetext{
* Contribution to the Topical Issue "Silicon and Siliconrelated Materials for Thermoelectricity", edited by Dario Narducci.

a e-mail: dario.narducci@unimib.it
}

papers demonstrated that phonon scattering at the outer roughened walls of silicon nanowires caused thermal conductance of single-crystalline silicon to drop by almost two orders of magnitude, with only a marginal impact onto its electrical conductivity. Since then the nanowire paradigm has been extended to a number of other materials, nanowires becoming a reference structure for thermoelectricity. Ways of manufacturing nanowires have quite evolved as well, especially for silicon where microelectronic technology could be used to obtain silicon nanowires with upscalable methods. Pennelli analyzes them in his review Top-down fabrication of silicon nanowire devices for thermoelectric applications, covering techniques that range from lithography to oxidation and highly anisotropic etching. Special attention is given to fabrication processes capable of robust industrialization. Redundant interconnectivity is discussed as a way to prevent local failures from impacting on the performances of the thermoelectric generator.

Also nanolayers have shown significant potentials for the reduction of silicon thermal conductivity. Their application to silicon is discussed by Neogi and Donadio in their research article Thermal transport in free-standing silicon membranes: influence of dimensional reduction and surface nanostructures. The authors provide an analysis of the phononic properties of Si nanomembranes, showing that also in 2D nanostructures the dimensional constraint by itself is not sufficient to significantly reduce the material thermal conductivity, surface roughness playing instead a key role. A different approach to the reduction of thermal conductivity is proposed by Dettori, Melis, and Colombo in their research paper $S i_{x} G e_{1-x}$ alloy as efficient phonon barrier in Ge-Si superlattices for thermoelectric applications. They show through atomistic simulations how the stoichiometry and the width of the SiGe layer in $\mathrm{Ge}_{\mid} \mathrm{Si}_{x} \mathrm{Ge}_{1-x}$ superlattices affect the overall thermal transport of the nanostructure. Interestingly, optimal (minimum) conductivity is found for $10 \% \leq x \leq$ $20 \%$ and for a 2:1 width ratio, namely both chemical and geometrical parameters admit an optimum. SiGe multilayers are also the focus of the review paper by Cecchi, 
Ferre Llin, Etzelstorfer, and Samarelli. In their Review of thermoelectric characterization techniques suitable for SiGe multilayer structures they discuss methods to reliably characterize Si-Ge thermoelectric heterostructures, where technical difficulties already arising in thin films are further exacerbated. Test boards that are normally required for in - and cross-plane thermoelectric characterization are described and critically discussed, also in view of the importance that a reliable, reproducible characterization has for nanostructures getting everyday closer to actual applications for on-chip cooling (as Peltier devices) and for energy integrated microharvesters (as Seebeck generators).

Use of nanograined materials as a way to reduce thermal conductivity is another classical approach to enhance thermoelectric properties that received however a new impetus by nanotechnology. The actual difficulty of controlling nanostructured silicon is covered by Petermann and co-workers in their research paper Thermoelectrics from silicon nanoparticles: the influence of native oxide where strategies to prevent oxidation while sintering doped $\mathrm{Si}$ nanoparticles are instanced. The possibility of improving thermoelectric efficiency by selectively reducing $\kappa$ over $\sigma$ is explored, finding that when the mass fraction of silicon dioxide is $<25 \%$ the combined effect of the lower electron mobility and thermal conductivity may anyway result in an increase of $Z T$, reaching 0.45 at $950{ }^{\circ} \mathrm{C}$.

Although the search for strategies to lower thermal conductivity has driven most of the late research effort in Si-based materials, dimensionally-constrained nanostructures may also serve the target of increasing silicon power factor. The paper that closes this Special Issue actually reports about the effect of nanosizing on the power factor. In his Prospects of low-dimensional and nanostructured silicon-based thermoelectric materials, Neophytou reviews possibilities of power factor enhancement in low-dimensional channels and in nanocrystalline materials. Enhanced power factors in ultra-narrow nanowires and ultra-thin layers are found to occur only upon suitable transport and confinement orientation, and only at an appropriate confinement length scale. In nanocrystalline silicon, instead, effective use of energy filtering that takes advantage of the inhomogeneity within the material is found to have the potential of large power factor improvements. Comparison of simulations to experimental results further support his findings.

In summary, I may anticipate that this Special Issue will not only provide the reader with an updated picture of the ongoing research on nanostructured silicon but will also further stimulate the effort needed to bring to the macroscopic scale the improved thermoelectric performances experimented and verified in nanostructures. All contributions, either experimental or computational, evidenced once again the extraordinary capability of silicon to reshape itself to meet new technological challenges - thermoelectricity being possibly the most recent but surely not the last one. My gratitude goes then to all authors to have built through their papers a clear confirmation of such silicon adaptability - and to all reviewers for their silent work, that made this Special Issue possible.

\section{References}

1. J.P. Heremans, M.S. Dresselhaus, L.E. Bell, D.T. Morelli, Nat. Nanotechnol. 8, 471 (2013)

2. G.J. Snyder, E.S. Toberer, Nat. Mater. 7, 105 (2008)

3. M.G. Kanatzidis, Chem. Mater. 22, 648 (2009)

4. A.J. Minnich, M.S. Dresselhaus, Z.F. Ren, G. Chen, Energy Environ. Sci. 2, 466 (2009)

5. A. Ioffe, Semiconductor Thermoelements and Thermoelectric Cooling (Infosearch Ltd., London, 1957)

6. A.I. Hochbaum, R.K. Chen, R.D. Delgado, W.J. Liang, E.C. Garnett, M. Najarian, A. Majumdar, P.D. Yang, Nature 451, 163 (2008)

7. A.I. Boukai, Y. Bunimovich, J. Tahir-Kheli, J.K. Yu, W.A. Goddard, J.R. Heath, Nature 451, 168 (2008) 